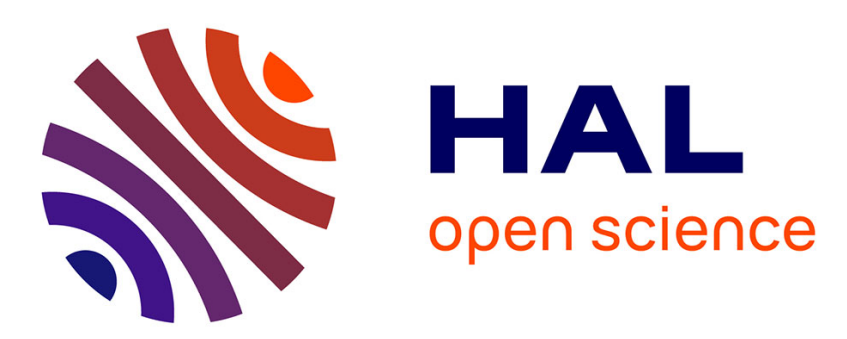

\title{
Évaluations différenciées des informations transmises par les cinq facteurs : effets de la dimension personnologique considérée et du genre de l'évaluateur Bernard Gangloff
}

\section{- To cite this version:}

Bernard Gangloff. Évaluations différenciées des informations transmises par les cinq facteurs : effets de la dimension personnologique considérée et du genre de l'évaluateur. Pratiques Psychologiques, 2011, 17 (1), pp.81 - 92. 10.1016/j.prps.2009.10.004 . hal-01693289

\section{HAL Id: hal-01693289 \\ https://hal.parisnanterre.fr/hal-01693289}

Submitted on 26 Jan 2018

HAL is a multi-disciplinary open access archive for the deposit and dissemination of scientific research documents, whether they are published or not. The documents may come from teaching and research institutions in France or abroad, or from public or private research centers.
L'archive ouverte pluridisciplinaire HAL, est destinée au dépôt et à la diffusion de documents scientifiques de niveau recherche, publiés ou non, émanant des établissements d'enseignement et de recherche français ou étrangers, des laboratoires publics ou privés. 


\title{
Évaluations différenciées des informations transmises par les cinq facteurs : effets de la dimension personnologique considérée et du genre de l'évaluateur
}

\author{
Differential evaluations of the Big Five information: Effects of the \\ information referent and of the appraiser gender \\ B. Gangloff
}

Département de psychologie, université de Rouen, 76821 Mont Saint-Aignan cedex, France

\begin{abstract}
Résumé
Nous avons proposé à 400 étudiants et étudiantes une liste d'adjectifs référant aux cinq grandes dimensions personnologiques (extraversion, amabilité, caractère consciencieux, stabilité émotionnelle et ouverture d'esprit), et nous leur avons indiqué qu'ils devraient rencontrer une personne dont ils ne sauraient rien hormis l'une de ces caractéristiques personnologiques résumée par l'un de ces adjectifs. La tâche de nos sujets était alors de cocher le degré d'utilité et le degré d'agréabilité de cette unique information. Les résultats mettent alors notamment en évidence, aussi bien hommes et femmes regroupés que par genre, que toutes les dimensions ne font pas l'objet des mêmes appréciations, ni en termes d'agréabilité ni en termes d'utilité. Ils montrent également que de nombreuses différences évaluatives existent entre hommes et femmes.
\end{abstract}

(C) 2009 Société française de psychologie. Publié par Elsevier Masson SAS. Tous droits réservés.

Mots clés : Big Five ; Personnalité ; Valeur ; Désirabilité sociale ; Utilité sociale

\begin{abstract}
We proposed to 400 students a list of adjectives refering to the five big personological dimensions (extraversion, kindness, conscientious character, emotional stability and open-mindedness), and we indicated them that they should meet someone they would not know anything of, except one of this personological characteristic summarized by one these adjectives. The task of our subjects was to check the degree of utility and the degree of desirability of this unique information. The results put notably in evidence, as well men and women
\end{abstract}

Adresse e-mail : bernard.gangloff@univ-rouen.fr. 
gathered together as separatly, that the dimensions are not the object of the same appreciations, neither in terms of desirability nor in terms of utility. They also show numerous evaluative differences between men and women.

(C) 2009 Société française de psychologie. Published by Elsevier Masson SAS. All rights reserved.

Keywords: Big Five; Personality; Value; Social desirability; Social utility

\section{Introduction}

Selon Beauvois (1990), lorsque l'on pense décrire un congénère, on réalise en fait moins une description, neutre, qu'une évaluation, en termes positifs ou négatifs, de la personne que l'on pense décrire. Nous attribuons en fait une valeur à cette personne ou plus exactement à ses différentes caractéristiques, valeur qui serait constituée de deux dimensions : la désirabilité et l'utilité (Beauvois et al., 1999). La désirabilité ferait alors référence au caractère agréable, attractif, plaisant de chacune des caractéristiques de l'objet évalué, et l'utilité à la faculté qu' aurait chacune de ces caractéristiques soit de permettre à l'objet de réaliser ses objectifs (Peeters, 1986, parle ainsi de «profitabilité pour soi »), soit de permettre à la société, prise globalement, de réaliser ses objectifs (Beauvois, 1995, parle ainsi «d'utilité sociale»). Il y aurait donc, du moins pour l'utilité, une différenciation sur le plan conceptuel. Pour autant, cette différenciation ne se retrouverait pas au niveau empirique : d'après une étude à paraitre réalisée par Cambon ${ }^{1}$, les caractéristiques profitables pour soi seraient également utiles à la société (les corrélations entre ces deux utilités variant entre 0,85 et 0,88 ). Quant aux rapports entre désirabilité et utilité, ils restent encore inconnus : Le Barbenchon et al. (2005) soulignent en effet que les résultats obtenus sur ces rapports sont contradictoires, certains mettant en évidence des corrélations positives, d'autres des corrélations négatives, et certains enfin l'absence de toute corrélation. Ces différentes données nous ont conduit à diverses interrogations et, partant, à une nouvelle opérationnalisation de l'étude de la valeur.

Notre première interrogation porte sur l'aspect social de la valeur. Lorsque l'on réalise une hétéro-évaluation, c'est-à-dire lorsque l'on évalue quelqu'un, c'est dans un objectif précis : on l'évalue pour soi, pour une organisation particulière, mais il est très délicat d'effectuer cette évaluation pour la société prise dans sa globalité ; ne serait-ce que parce que toute société globale est multiforme, disparate, et poursuit des objectifs contradictoires selon les sous-groupes qui la constituent. L'utilité (comme la désirabilité) est ainsi fonction d'objectifs particuliers et ne peut être examinée que relativement à ces objectifs. Par exemple, un recruteur se demandera si tel trait personnologique (si tel positionnement sur un trait personnologique) est ou non utile à l'entreprise contractante, au poste à pourvoir... et non de manière générale, non dans l'absolu. Tout examen de la valeur devant donc nécessairement, sur le plan opérationnel, être contextualisé, cela signifie que le terme de «social» (désirabilité sociale, utilité sociale) nous semble, du fait de sa décontextualisation, trop général, trop polysémique, pour ne pas donner lieu à des résultats très divers, voire contradictoires. Nous avons donc décidé d'examiner ici l'attribution sociale de la valeur d'une personne, mais en précisant ce «social» et plus précisément en le concrétisant par une autre personne. En d'autres termes, nous nous sommes interrogés sur la valeur que la personne $\mathrm{X}$, objet d'évaluation, présentait pour la personne Y, évaluateur de X.

\footnotetext{
${ }^{1}$ Information que nous a fournie Cambon.
} 
Un autre point d'interrogation a trait aux outils de mesure de la valeur : ces outils renvoient souvent à des critères bien particuliers dont la validité peut alors être questionnée. Le Barbenchon et al. (2005) demandent ainsi à leurs sujets d'indiquer: pour la désirabilité si la cible «a tout pour être aimé » (vs «n'a rien pour être aimé ») et «a beaucoup d'amis » (vs «a peu d'amis »); et pour l'utilité si la cible «a tout pour réussir sa vie professionnelle ( vie professionnelle ») et «a un fort salaire» (vs «a un faible salaire»). Ajoutons, concernant en particulier ces deux échelles de mesure de l'utilité, que les auteurs soulignent (p. 312) que «ces deux échelles sont les plus utilisées pour mesurer l'utilité sociale ». Or évaluer l'utilité sociale à partir de la réussite professionnelle et du salaire peut sembler réducteur. D'abord parce que l'utilité dite «sociale» ne se résume pas au domaine professionnel. Ensuite, même en restant sur le plan professionnel, parce que s'il est certes possible que, dans les pays anglo-saxons, le salaire constitue une preuve d'utilité (utilité professionnelle donc), il faut se rappeler que les pays anglo-saxons ne représentent pas l'ensemble de la planète. Certes, aussi bien Beauvois que les auteurs travaillant sur son modèle spécifient qu'ils prennent l'utilité dans un sens économique (ainsi Beauvois, 1995, p. 378 ; ou encore Le Barbenchon et al., 2005, p. 309); mais outre que la valeur d'échange d'un bien, valeur qui peut prendre la forme de salaire, est loin de refléter systématiquement sa valeur économique, notamment en raison de la mondialisation (mon tailleur, qui soustraite en Asie, aura beau être riche, et bien plus riche que mon boulanger, les habitants de mon quartier considèrent qu'il leur est bien moins utile que celui qui pétrit de ses mains leur pain quotidien), il nous semble incorrect de parler d'utilité «sociale» tout en négligeant une autre de ses composantes, notamment sa valeur d'usage (si le salaire était le seul critère d'utilité sociale, alors les activités bénévoles seraient socialement totalement inutiles). Enfin, et à nouveau sur l'utilité sociale, il nous semble réducteur de ne considérer que les conduites utiles versus inutiles et d'oublier les conduites nuisibles (que Beauvois, 1976, appelait conduites «perverses »). Or «avoir tout pour réussir sa vie» nous semble pouvoir aussi bien être l'opposé de «n'avoir rien pour réussir sa vie» que de «avoir tout pour échouer sa vie». Et l'on pourrait tenir des raisonnements similaires pour la désirabilité (ne peut-on par exemple opposer «a beaucoup d'amis » à «a beaucoup d'ennemis » ?). Cela nous a donc conduit à utiliser ici de nouveaux critères de mesure de l'utilité et de la désirabilité, en l'occurrence des critères d'ordre personnologique.

Par ailleurs, lorsque nous évaluons quelqu'un sur des dimensions personnologiques, c'est parce que nous considèrons qu'obtenir une information sur le positionnement de cette personne relativement à ces dimensions nous est, compte tenu de notre objectif, utile. Prenons pour illustration le cas d'un consultant en recrutement. Il commencera, par enquête, à chercher des informations sur les traits et positionnements utiles pour l'entreprise contractante (et il va apprendre, par exemple, que les traits «ouverture d'esprit» et «dynamisme» sont utiles à cette entreprise, et également apprendre qu'un «bon candidat» doit se situer aux niveaux $\mathrm{X}$ et $\mathrm{Y}$ sur ces traits); puis il fera passer aux candidats un inventaire de personnalité constitué des deux traits retenus, les réponses à ces traits lui permettant de connaître le positionnement des candidats, c'est-à-dire que ces réponses constitueront des informations utiles; et au terme de sa mission, il retiendra les candidats dont le positionnement correspond au positionnement attendu, c'est-à-dire au positionnement utile à l'entreprise. Tout cela, nous le savons tous. Nous le savons, mais exprimé de cette façon, cela prend une signification particulière. Cela signifie que ce que recherche le recruteur, ce sont principalement des informations; des informations sur des utilités, inutilités et nuisibilités certes, mais qui n'en sont pas moins des informations. Ce qu'examine ainsi d'abord un recruteur, c'est l'utilité de la connaissance de traits et de la connaissance du positionnement des candidats sur des traits. Les premières utilités 
Distribution des questionnaires dans les groupes et répartition des sujets selon leur genre.

Questionnaire 1 (20 adjectifs)

Questionnaire 2 (20 adjectifs)

Questionnaire 3 (20 adjectifs)

Questionnaire 4 (20 adjectifs)

Questionnaire 5 (20 adjectifs)

Total : 100 adjectifs
40 hommes et 40 femmes

40 hommes et 40 femmes

40 hommes et 40 femmes

40 hommes et 40 femmes

40 hommes et 40 femmes

Total : 200 hommes et 200 femmes

portent donc sur des informations : ces informations me sont-elles utiles, inutiles ou nuisibles? Et ce n'est qu'en conclusion à la procédure de recrutement que le recruteur se demandera si le positionnement des candidats est bon, c'est-à-dire est utile, inutile ou nuisible à l'entreprise contractante ${ }^{2}$.

Nous avons donc ici souhaité mesurer la valeur, non pas de caractéristiques personnologiques, mais d'informations sur des caractéristiques personnologiques.

\section{Méthode}

Nous avons utilisé 100 adjectifs référant aux cinq dimensions de l'Alter Ego (test de la famille du Big Five) à raison de 20 adjectifs par dimension, soit dix adjectifs pour chacun des deux pôles d'une dimension (ainsi, pour le pôle positif de la dimension «caractère consciencieux », nous avons les dix adjectifs suivants : consciencieux, méticuleux, pointilleux, persévérant, déterminé, acharné, minutieux, réfléchi, ordonné, tenace. Et pour le pôle négatif de cette même dimension, nous avons les adjectifs opposés : «peu consciencieux », «peu méticuleux », etc.) $)^{3}$.

L'application de ces 100 adjectifs en un seul questionnaire rendant délicate son application à une seule population, ces 100 adjectifs ont été répartis aléatoirement dans cinq questionnaires à raison de 20 adjectifs par questionnaire (dix avec un pôle positif et dix avec un pôle négatif). Ces questionnaires ont ensuite été répartis sur 400 étudiants et étudiantes de diverses disciplines (sauf psychologie, afin d'écarter les étudiants ayant pu avoir connaissance des travaux de psychologie portant sur la valeur) répartis en cinq groupes, chaque étudiant répondant individuellement au questionnaire qui lui était proposé. La distribution de ces cinq questionnaires dans ces cinq groupes est indiquée dans le Tableau 1.

Nous avons par ailleurs voulu examiner non pas la valeur de chacun des adjectifs mais la valeur des informations sur ces adjectifs. Quant aux critères d'évaluation de cette valeur, elle consistait à demander si l'information fournie était « agréable, indifférente ou désagréable » (pour la désirabilité) et «utile, sans intérêt ou gênante» (pour l'utilité) ${ }^{4}$. La consigne était ainsi la suivante :

\footnotetext{
${ }^{2}$ Et un raisonnement similaire pourrait être tenu au sujet de la désirabilité.

${ }^{3}$ Ces 100 adjectifs, qui sont présentés en Annexe 1 en les mettant en relation avec les cinq dimensions du Big Five, ont été validés par rapport à la version française de l'Alter Ego par Pasquier en 2007 (Pasquier, 2007), avec notamment l'obtention, selon la dimension et le pôle : de coefficents alpha variant de 0,75 à 0,90 et d'indices de régression linaire compris entre 0,49 et 0,88 .

4 Précisons, pour répondre à la remarque d'un lecteur, que considérant «utile» et «gênant » en opposition, il nous a semblé plus pertinent de construire une seule échelle d'utilité (échelle à deux pôles opposés avec possibilité d'une réponse centrale) plutôt que deux échelles (utile/inutile et utile/nuisible - ou utile/gênant). Le même raisonnement vaut aussi pour la désirabilité.
} 
«Dans le questionnaire suivant, il vous est demandé d'imaginer que vous allez bientôt être mis en contact avec une personne que vous ne connaissez pas. Vous ne savez pas non plus s'il s'agit d'un garçon ou d'une fille, si vous allez rencontrer cette personne dans un cadre professionnel, amical ou autre, etc. En fait, vous n'avez qu'une seule information sur cette personne. Vous devrez alors indiquer, d'une part, si cette information vous est agréable, ou indifférente ou désagréable, en expliquant ensuite pourquoi, et d'autre part, si cette information vous est utile, ou sans intérêt, ou si vous préfèreriez ne pas avoir cette information car elle vous dérange trop, en expliquant aussi ensuite pourquoi $\gg$ (un exemple d'item complet est donné en Annexe 2).

Chacun des cinq questionnaires comprenant 20 adjectifs auxquels 80 étudiants répondent, nous avons ainsi un total de 1600 réponses d'utilité et 1600 réponses de désirabilité par questionnaire. En termes de dimension, puisque chaque dimension était caractérisée par 20 adjectifs, nous avons également 1600 réponses d'utilité et 1600 réponses de désirabilité pour chacune des cinq dimensions ( 800 sur les pôles positifs et 800 sur les pôles négatifs pour chacune de nos deux variables dépendantes), soit au total 8000 réponses d'utilité et 8000 réponses de désirabilité pour l'ensemble des cinq dimensions (est reportée en Annexe 3 une illustration des données obtenues pour les jugements d'agréabilité sur les dimensions extraversion et ouverture d'esprit dans leur pôle positif).

Les résultats obtenus, c'est-à-dire les choix des répondants, ont été analysés dimension par dimension ${ }^{5}$, d'une part, hommes et femmes confondus et, d'autre part, par genre. Nous avons ainsi examiné, pour chaque item, la répartition de ces choix dans les trois alternatives de réponses proposées référant à chacune des deux dimensions étudiées (désirabilité et utilité) et calculé (par $\mathrm{X}^{2}$ à systématiquement 1 ddl) l'éventuelle inégale répartition de ces choix interdimensions personnologiques en comparant, sur chacun des pôles extrêmes (A+, A-,U+ et $\left.\mathrm{U}_{-}\right)$, les éventuelles différences de classements entre les cinq dimensions $^{6}$. Soit, à titre d'illustration, la comparaison, chez les femmes, des jugements extrêmes d'agréabilité à la dimension «extraversion» pôle positif par rapport aux jugements d'agréabilité à la dimension «ouverture d'esprit» pôle positif (Annexe 3): nous y observons, pour les réponses «agréable», 182 réponses pour l'extraversion et 233 pour l'extraversion, soit un $\mathrm{X}^{2}$ à 1 ddl de 6,27 signifiant qu'une information faisant état d'ouverture d'esprit est jugée significativement plus agréable (à $p=0,01)$ qu'une information révélant de l'extraversion.

\footnotetext{
${ }^{5}$ Il est bien évident que les psychologues utilisant des inventaires de personnalité interprètent les réponses fournies non seulement dimension par dimension mais également dans leurs conjonctions (de même qu'ils examinent ces données parallèlement à des informations portant sur d'autres caractéristiques : compétences issues des diplômes et de l'expérience... .). Rappelons alors que notre objectif n'est pas de reproduire une situation d'évaluation dans sa totalité et dans sa complexité, d'où notre choix de restreindre ici notre analyse aux fondements évaluatifs, c'est-à-dire à la valeur des informations fournies sur chaque dimension prise isolément.

${ }^{6}$ La possibilité d'une réponse centrale a essentiellement été proposée pour éviter à nos sujets de se sentir « contraints » à un choix parfois délicat entre deux extrêmes. Aussi, outre que ces réponses centrales peuvent donner lieu à de multiples interprétations (Caprara et al., 1997, p. 32) rendant leur traitement bien hasardeux, il est évident que, eu égard à notre objectif, les données les plus pertinentes concernent les choix extrêmes. Nous avons donc choisi de ne traiter que ces derniers (rappelons que notre objectif était de mesurer des préférences, c'est-à-dire s'il est plus agréable ou plus désagréable - et plus utile ou plus gênant - d'obtenir une information sur une dimension personnologique $\mathrm{X}$ que sur une dimension personnologique Y, indépendanmment des déterminants de ces préférences, et notamment indépendamment du fait que ces préférences, que ces choix exrêmes donc, peuvent parfois résulter des fréquences des réponses intermédiaires).
} 
Tableau 2

Résumé des différences significatives interdimensions pour l'agréabilité (en italiques figurent les résultats obtenus seulement chez les femmes ou seulement chez les hommes).

\begin{tabular}{|c|c|c|c|c|}
\hline & A+ pôles positifs & $\mathrm{A}+$ pôles négatifs & A- pôles positifs & A- pôles négatifs \\
\hline $\begin{array}{l}\text { E/O (extraversion/ouverture } \\
\text { d'esprit) }\end{array}$ & $\mathrm{O}>\mathrm{E}$ & $\mathrm{E}>\mathrm{O}$ & $\mathrm{E}>\mathrm{O}$ & $O>E($ chez $F)$ \\
\hline $\begin{array}{l}\text { E/C (extraversion/caractère } \\
\text { consciencieux) }\end{array}$ & & & $E>C($ chez $F)$ & $C>E($ chez $F)$ \\
\hline E/G (extraversion/amabilité) & $\mathrm{G}>\mathrm{E}$ & $E>G$ & $E>G$ & $\mathrm{G}>\mathrm{E}$ \\
\hline $\begin{array}{l}\text { E/N (extraversion/stabilité } \\
\text { émotionelle) }\end{array}$ & $\mathrm{N}>\mathrm{E}$ & $E>N($ chez $F)$ & $\mathrm{E}>\mathrm{N}$ & $\mathrm{N}>\mathrm{E}$ \\
\hline $\begin{array}{l}\mathrm{O} / \mathrm{C} \text { (ouverture d'esprit /caractère } \\
\text { consciencieux) }\end{array}$ & $\mathrm{O}>\mathrm{C}$ & $C>O($ chez $F)$ & $\mathrm{C}>\mathrm{O}$ & \\
\hline $\mathrm{O} / \mathrm{G}$ (ouverture d'esprit/amabilité & $G>O($ chez $F)$ & & & $\mathrm{G}>\mathrm{O}$ \\
\hline $\begin{array}{l}\mathrm{O} / \mathrm{N} \text { (ouverture d'esprit/stabilité } \\
\text { émotionelle) }\end{array}$ & & & $O>N($ chez $F)$ & $N>O($ chez $H)$ \\
\hline $\begin{array}{l}\text { C/G (caractère } \\
\text { consciencieux/amabilité) }\end{array}$ & $\mathrm{G}>\mathrm{C}$ & $\mathrm{C}>\mathrm{G}$ & $\mathrm{C}>\mathrm{G}$ & $\mathrm{G}>\mathrm{C}$ \\
\hline $\begin{array}{l}\mathrm{C} / \mathrm{N} \text { (caractère } \\
\text { consciencieux/stabilité } \\
\text { émotionelle) }\end{array}$ & $\mathrm{N}>\mathrm{C}$ & $C>N($ chez $F)$ & $\mathrm{C}>\mathrm{N}$ & $\mathrm{N}>\mathrm{C}$ \\
\hline $\begin{array}{l}\text { G/N (amabilité/stabilité } \\
\text { émotionelle) }\end{array}$ & & & $G>N($ chez $F)$ & \\
\hline
\end{tabular}

\section{Résultats}

\subsection{Pour l'agréabilité}

La lecture du Tableau 2, qui résume les différences significatives (avec $p<0,05$ ) obtenues sur le plan de l'agréabilité, permet d'aboutir à différents constats :

- en agréabilité + (informations jugées agréables) et sur le pôle positif de chacune des cinq dimensions, on remarque, aussi bien chez les hommes que chez les femmes, que les informations sur les dimensions ouverture d'esprit, amabilité et stabilité émotionnelle sont considérées comme plus agréables que celles portant sur les dimensions extraversion ou caractère consciencieux (soit les inégalités suivantes : ouverture d'esprit, amabilité et stabilité émotionnelle supérieures à extraversion et caractère consciencieux $)^{7}$. On remarque par ailleurs, mais seulement chez les femmes, que les informations sur la dimension amabilité sont jugées plus agréables que celles sur l'ouverture d'esprit (amabilité supérieure à ouverture d'esprit);

- en agréabilité - (informations jugées désagréables) et toujours sur le pôle positif des dimensions, les hiérarchies suivantes sont constatées de manière systématique (c'est-à-dire chez les hommes comme chez les femmes) : extraversion et caractère consciencieux supérieurs à ouverture d'esprit, amabilité et stabilité émotionnelle ${ }^{8}$. D'autre part, mais à nouveau uniquement chez les femmes, ouverture d'esprit et amabilité supérieurs à stabilité émotionnelle, et extraversion supérieure à caractère consciencieux ;

\footnotetext{
${ }^{7}$ En annexe 3, à titre d'exemple, les comparaisons des réponses « agréable » pour extraversion/ouverture d'esprit.

${ }^{8}$ En annexe 3, à titre d'exemple, les comparaisons des réponses « désagréable » pour extraversion/ouverture d'esprit.
} 
Tableau 3

Résumé des différences significatives interdimensions pour l'utilité (en italiques figurent les résultats obtenus seulement chez les femmes ou seulement chez les hommes).

\begin{tabular}{|c|c|c|c|c|}
\hline & U+ pôles positifs & U+ pôles négatifs & U- pôles positifs & U- pôles négatifs \\
\hline $\begin{array}{l}\text { E/O (extraversion/ouverture } \\
\text { d'esprit) }\end{array}$ & & $\mathrm{E}>\mathrm{O}$ & $E>O($ chez $F)$ & $\mathrm{O}>\mathrm{E}$ \\
\hline $\begin{array}{l}\text { E/C }(\text { extraversion/caractère } \\
\text { consciencieux })\end{array}$ & & $E>C($ chez $F)$ & & \\
\hline E/G (extraversion/amabilité) & $\mathrm{G}>\mathrm{E}$ & & $E>G(\operatorname{chez} F)$ & $\mathrm{G}>\mathrm{E}$ \\
\hline $\begin{array}{l}\text { E/N (extraversion/stabilité } \\
\text { émotionelle) }\end{array}$ & & $N>E($ chez $H)$ & $E>N($ chez $F)$ & $N>E($ chez $F)$ \\
\hline $\begin{array}{l}\mathrm{O} / \mathrm{C} \text { (ouverture d'esprit/caractère } \\
\text { consciencieux) }\end{array}$ & $O>C($ chez $F)$ & & $\mathrm{C}>\mathrm{O}$ & $\mathrm{O}>\mathrm{C}$ \\
\hline $\mathrm{O} / \mathrm{G}$ (ouverture d'esprit/amabilité & $G>O($ chez $F)$ & $\mathrm{G}>\mathrm{O}$ & & \\
\hline $\begin{array}{l}\mathrm{O} / \mathrm{N} \text { (ouverture d'esprit/stabilité } \\
\text { émotionelle) }\end{array}$ & & $\mathrm{N}>\mathrm{O}$ & & $O>N($ chez $H)$ \\
\hline $\begin{array}{l}\text { C/G (caractère } \\
\text { consciencieux/amabilité) }\end{array}$ & $\mathrm{G}>\mathrm{C}$ & $G>C($ chez $H)$ & $C>G($ chez $H)$ & $G>C($ chez $H)$ \\
\hline $\begin{array}{l}\mathrm{C} / \mathrm{N} \text { (caractère } \\
\text { consciencieux/stabilité } \\
\text { émotionelle) }\end{array}$ & $N>C($ chez $F)$ & $\mathrm{N}>\mathrm{C}$ & $\mathrm{C}>\mathrm{N}$ & \\
\hline $\begin{array}{l}\mathrm{G} / \mathrm{N} \text { (amabilité/stabilité } \\
\text { émotionelle) }\end{array}$ & $G>N($ chez $F)$ & & $G>N($ chez $F)$ & $G>N($ chez $H)$ \\
\hline
\end{tabular}

- en agréabilité +, maintenant sur le pôle négatif de chacune des cinq dimensions, les hiérarchies suivantes sont obtenues : extraversion et caractère consciencieux supérieurs à amabilité ; extraversion supérieure à ouverture d'esprit. Par ailleurs, toujours uniquement chez les femmes, les informations sur l'extraversion sont jugées plus agréables que celles portant sur le caractère consciencieux ou sur la stabilité émotionnelle (extraversion supérieure à caractère consciencieux et stabilité émotionnelle), de même que celles sur le caractère consciencieux sont dites plus agréables que celles renvoyant à l'ouverture d'esprit (caractère consciencieux supérieur à ouverture d'esprit);

- en agréabilité - (et à nouveau sur le pôle négatif des dimensions), nous obtenons : amabilité supérieure à extraversion, ouverture d'esprit et caractère consciencieux ; stabilité émotionnelle supérieure à extraversion et caractère consciencieux. On observe enfin également ouverture d'esprit et caractère consciencieux supérieurs à extraversion chez les femmes, et stabilité émotionnelle supérieure à ouverture d'esprit chez les hommes.

\subsection{Pour l'utilité}

Les différences significatives (toujours avec $p<0,05$ ) ayant trait aux jugements d'utilité sont consignées dans le Tableau 3. Elles mettent en évidence les aspects suivants :

- en utilité + (informations jugées utiles) et sur le pôle positif de chacune des cinq dimensions, on constate de manière systématique que les informations sur l'amabilité sont plus utiles que celles sur l'extraversion ou sur le caractère consciencieux (soit amabilité supérieure à extraversion et caractère consciencieux). Mais des différences supplémentaires sont aussi mises en évidence chez les femmes : amabilité supérieure à ouverture d'esprit et stabilité émotionnelle, ouverture d'esprit et stabilité émotionnelle supérieures à caractère consciencieux ; 
- en utilité - (informations jugées nuisibles) et toujours sur le pôle positif des dimensions, ce sont deux inégalités qui sont observées : caractère consciencieux supérieur à ouverture d'esprit et stabilité émotionnelle. Enfin, uniquement chez les femmes, nous constatons quatre différences complémentaires (extraversion supérieure à ouverture d'esprit, amabilité et stabilité émotionnelle, et amabilité supérieure à stabilité émotionnelle), et chez les hommes une dernière différence particulière (caractère consciencieux supérieur à amabilité) ;

- en utilité +, maintenant sur le pôle négatif de chacune des cinq dimensions, quatre différences sont systématiques : extraversion, amabilité et stabilité émotionnelle supérieures à ouverture d'esprit, et stabilité émotionnelle supérieure à caractère consciencieux. D'autre part, spécifiquement chez les femmes, extraversion supérieure à caractère consciencieux; et spécifiquement chez les hommes, amabilité supérieure à caractère consciencieux, ainsi que stabilité émotionnelle supérieure à extraversion;

- enfin, en utilité - (et une nouvelle fois sur le pôle négatif des dimensions), les hiérarchies systématiques sont les suivantes : ouverture d'esprit et amabilité supérieures à extraversion, et ouverture d'esprit supérieure à caractère consciencieux. Uniquement chez les femmes, on obtient aussi stabilité émotionnelle supérieure à extraversion. Les hommes font enfin également état de classements qui leurs sont particuliers, avec des informations qui sont considérées comme plus nuisibles quand elles portent sur l'ouverture d'esprit et sur l'amabilité que lorsqu'elles concernent la stabilité émotionnelle (ouverture d'esprit et amabilité supérieures à stabilité émotionnelle), et également davantage de réponses «nuisible » sur l'amabilité que sur le caractère consciencieux (amabilité supérieure à caractère consciencieux).

\section{Discussion}

Il a été montré, dans une récente étude (Gangloff et Mazilescu, 2007) utilisant la même procédure mais avec d'autres adjectifs cibles que ceux issus du Big Five, que les informations personnologiques sont pratiquement toujours jugées utiles, quelle que soit l'éventuelle intensité de désagréabilité qu'elles véhiculent: mêmes si les informations sont désagréables, les répondants considèrent pratiquement toujours qu'elles sont utiles et souhaitent les obtenir. Dans un autre travail, Auzoult et Abdellaoui (2008) ont aussi, plus largement, constaté que les jugements d'agréabilité pouvaient être déconnectés des jugements d'utilité. L'examen des réponses aux questions ouvertes permet parfois de mieux comprendre cette déconnexion, essentiellement constatée pour les jugements portant sur les pôles négatifs des dimensions ${ }^{9}$. On remarque ainsi que c'est un souhait d'anticipation qui explique les attributions d'utilité face à une information par ailleurs jugée désagréable. Ainsi l'information «turbulent» (de la dimension stabilité émotionnelle) est considérée comme désagréable ( «j'aime les gens posés ») mais utile ( «je vais anticiper une situation délicate»). Et c'est a contrario un rejet de cette anticipation qui explique certains jugements d'utilité négative, de gêne, de souhait de ne pas avoir obtenir une information, lorsque celle-ci est considérée comme désagréable. Ainsi l'information «intolérant» (de la dimension amabilité) est désagréable ( «je ne supporte pas ») et gênante ( «je vais me fixer sur cette information et ça m'empêchera de m'intéresser aux autres traits de caractère de cette personne »); l'information

\footnotetext{
${ }^{9}$ Les déconnexions sont beaucoup plus rares pour les informations renvoyant aux pôles positifs des dimensions. Ainsi l'information «large d'esprit», qui réfère au pôle positif de la dimension «ouverture d'esprit », est considérée comme aussi bien agréable qu'utile (avec par exemple comme raisons respectives avancées : «je suis quelqu'un de bavard, donc en apprenant cela je sais que je vais pouvoir discuter sur plein de sujets » et «ça me donnera hâte de rencontrer cette personne »).
} 
« ombrageux » (de la dimension stabilité émotionnelle) est désagréable («je vais m'embêter, elle ne va pas être intéressante ») et gênante ( «parce que je sais que je vais m'embêter; je ne l'aurais pas su, je me serais fait mon opinion moi-même») etc.

On observe également ici que les jugements portés sur ces informations, qu'il s'agisse des jugements d'agréabilité ou de ceux d'utilité, varient selon la dimension considérée. Pour l'agréabilité, nous remarquons par exemple que l'amabilité est systématiquement différenciée de l'extraversion et du caractère consciencieux : apprendre que quelqu' un est « aimable » est toujours une information plus agréable que celle d'apprendre que quelqu'un est extraverti ou est consciencieux; corollairement, il est plus désagréable d'apprendre que quelqu'un n'est pas aimable que d'apprendre qu'il est introverti ou peu consciencieux. De même, au niveau de l'utilité, il est considéré plus utile de savoir que quelqu'un est aimable que de savoir qu'il est extraverti ou consciencieux, et corollairement il est plus gênant d'apprendre que quelqu'un est peu aimable que de savoir qu'il est introverti ou (pour les hommes) qu'il est peu consciencieux. Si le fait de ces hiérarchies nous semble pouvoir être considéré comme général, la composition de ces hiérarchies peut quant à elle relever de la conjoncture et provenir de la consigne diffusée à nos sujets : il s'agissait pour eux de porter des jugements vis-à-vis d'une personne qu'ils devaient rencontrer sans que soit précisé l'objectif de cette rencontre. Il est alors fort possible que si le contexte avait été différent, d'autres résultats eussent été obtenus (par exemple, si cette rencontre avait eu pour objectif de réaliser, de manière coopérative, une tâche minutieuse, le caractère consciencieux aurait sans doute pris davantage d'importance).

On remarque également de nombreuses différences de jugements entre hommes et femmes. Ainsi, pour l'agréabilité, sur 30 comparaisons significatives, un tiers est spécifique soit aux hommes soit (plus généralement) aux femmes; et pour l'utilité, ce pourcentage passe à $59 \%$ (sur 27 différences significatives, 16 ne sont le fait que des hommes ou que des femmes). Sans entrer dans le détail de la nature des dimensions jugées plus agréables (ou plus utiles) que d'autres de façon différenciée chez les hommes ou chez les femmes, nous remarquerons alors essentiellement que les femmes réalisent davantage de différenciations que les hommes, tant sur le plan de l'agréabilité (neuf sont spécifiques aux femmes contre une seule pour les hommes) que de l'utilité (respectivement dix et six). L'une des conséquences, par exemple, en situation d'évaluation d'autrui (dans le cadre d'un bilan de compétences, d'une orientation professionnelle, d'un recrutement...), est que les hommes et les femmes n'examineront pas avec la même finesse discriminative les différentes informations dont ils pourront disposer (les femmes discriminant plus fréquemment que les hommes, elles attacheront des importances plus différenciées à ces informations que ne le feront les hommes). Et cela se traduira bien évidemment notamment sur le plan du contenu des diagnostics réalisés.

On ne peut pas exclure que les résultats de cette étude, réalisée sur un public d'étudiants, traduisent, ou tout au moins soient susceptibles de traduire, nombre des conduites observables dans de réelles situations de recrutement mené par des professionnels. À ce titre, nous espérons que cette mise en évidence du caractère relatif dont sont traitées les informations fournies à des inventaires de personnalité aidera les recruteurs à affiner leur prise de conscience de ce traitement, leur permettant ainsi un meilleur accomplissement de leurs missions.

\section{Conclusion}

Nous avons, dans cette étude, voulu connaître les jugements d'agréabilité et d'utilité portés non sur des caractéristiques personnologiques mais sur des informations ayant trait à ces caractéristiques personnologiques : il ne s'agissait pas de juger si telle caractéristique de personnalité était 
agréable, désagréable, utile ou gênante, mais de juger si l'information indiquant qu'un individu était porteur de cette caractéristique personnologique (ou de cette caractéristique normative ${ }^{10}$ ), était agréable, désagréable, utile ou gênante ${ }^{11}$.

Il est alors constaté que les réponses obtenues varient selon les dimensions et que (par exemple) une information sur la dimension amabilité fait toujours l'objet d'appréciations plus intenses (que ce soit en agréabilité ou en utilité) qu'une information sur les dimensions extraversion ou caractère consciencieux. Mais comme nous l'avons indiqué dans notre discussion, il est possible que les hiérarchies obtenues dans la présente étude, que nous avons voulue exploratoire, soient fonction de la consigne utilisée et qu'une autre consigne (par exemple rencontrer quelqu'un afin de réaliser de manière coopérative une tâche minutieuse) eût conduit à des hiérarchies différentes. Cela signifie qu'il convient maintenant, par d'autres études, d'introduire des contextes afin d'examiner notamment les éventuels effets de l'objectif de la rencontre proposée.

Nous remarquons également qu'hommes et femmes réagissent différemment, avec en particulier davantage de discriminations interdimensions chez les femmes. Du fait de la complexité de la variable genre, nous nous sommes abstenus d'en avancer une explication. Nous avons cependant, dans notre discussion, évoqué quelques implications pratiques de ce constat en soulignant l'importance du genre de l'évaluateur au niveau des diagnostics réalisés en situations d'évaluation d'autrui. D'autres études sont donc également maintenant nécessaires pour préciser la concrétisation de ces implications. . .

\section{Annexe 1. Les 100 adjectifs utilisés avec leur mise en rapport avec les cinq dimensions du Big Five}

\begin{tabular}{|c|c|c|c|c|c|c|c|}
\hline N- & Ombrageux & $\mathrm{N}-$ & Vulnérable & $\mathrm{N}+$ & Equilibré & $\mathrm{C}-$ & Peu combatif \\
\hline $\mathrm{N}-$ & Turbulent & $\mathrm{G}+$ & Agréable & $\mathrm{E}+$ & Pugnace & $\mathrm{C}+$ & Ordonné \\
\hline $\mathrm{N}+$ & Maître de soi & $\mathrm{O}+$ & Lecteur & $\mathrm{O}-$ & Étroit d'esprit & $\mathrm{C}+$ & Tenace \\
\hline N- & Agité & $\mathrm{O}-$ & Traditionaliste & $\mathrm{G}+$ & Courtois & $\mathrm{E}-$ & Silencieux \\
\hline $\mathrm{C}-$ & Indolent & $\mathrm{O}-$ & Inculte & $\mathrm{C}+$ & Déterminé & $\mathrm{O}+$ & Ouvert \\
\hline $\mathrm{G}-$ & Individualiste & $\mathrm{G}-$ & Insensible & E+ & Décidé & $\mathrm{G}+$ & Sincère \\
\hline $\mathrm{N}-$ & Irritable & $\mathrm{E}+$ & Sûr de soi & $\mathrm{O}+$ & Progressiste & $\mathrm{O}+$ & Novateur \\
\hline $\mathrm{C}-$ & Velléitaire & $\mathrm{E}-$ & En retrait & $\mathrm{C}-$ & Irresponsable & $\mathrm{G}+$ & Soumis \\
\hline $\mathrm{O}-$ & Fermé & $\mathrm{C}+$ & Méticuleux & $\mathrm{C}+$ & Acharné & $\mathrm{O}+$ & Curieux \\
\hline $\mathrm{C}$ & Consciencieux & $\mathrm{G}+$ & Accommodant & $\mathrm{G}+$ & Conciliant & $\mathrm{E}+$ & Direct \\
\hline $\mathrm{N}-$ & Énervé & $\mathrm{C}-$ & Désordonné & $\mathrm{C}+$ & Minutieux & $\mathrm{G}-$ & Intolérant \\
\hline $\mathrm{E}+$ & Dominateur & $\mathrm{E}-$ & Introverti & $\mathrm{N}-$ & Inquiet & $\mathrm{O}+$ & Large d'esprit \\
\hline $\mathrm{O}-$ & Normatif & $\mathrm{E}-$ & Discret & $\mathrm{G}-$ & Égocentrique & $\mathrm{N}+$ & Calme \\
\hline $\mathrm{N}+$ & Solide & $\mathrm{N}+$ & Décontracté & $\mathrm{G}-$ & Soupçonneux & $\mathrm{O}+$ & Avide de culture \\
\hline $\mathrm{N}+$ & Contrôlé & $\mathrm{O}-$ & Sectaire & $\mathrm{G}+$ & Humaniste & $\mathrm{G}-$ & Méfiant \\
\hline $\mathrm{E}-$ & Renfermé & $\mathrm{G}+$ & Sociable & $\mathrm{O}+$ & Cérébral & $\mathrm{E}-$ & Réservé \\
\hline $\mathrm{G}-$ & Déplaisant & $\mathrm{O}+$ & Cultivé & $\mathrm{C}+$ & Réfléchi & $\mathrm{E}+$ & Persuasif \\
\hline E+ & Ambitieux & $\mathrm{G}+$ & Coopératif & $\mathrm{N}+$ & Sang-froid & $\mathrm{E}-$ & Effacé \\
\hline $\mathrm{C}-$ & Expéditif & $\mathrm{N}-$ & Anxieux & $\mathrm{O}-$ & Ignare & $\mathrm{C}-$ & Approximatif \\
\hline
\end{tabular}

\footnotetext{
${ }^{10}$ D'après les résultats d'une récente étude (Gangloff, 2003), on peut en effet se demander si les cinq dimensions du Big Five ne doivent pas se voir attribuer le statut de normes sociales plutôt que celui de dimensions personnologiques.

11 Précisons par ailleurs que nous avons pris soin, notamment par la typographie de notre questionnaire, de bien souligner qu'il s'agissait de juger des informations et non les individus sur lesquels portaient ces informations. Et l'examen des réponses aux questions ouvertes, dont nous avons, dans la discussion, fourni quelques illustrations, confirme le caractère effectif de cette procédure.
} 
Annexe 1 (Suite)

\begin{tabular}{llllllll}
\hline G- & Asocial & $\mathrm{C}-$ & Bâcleur & $\mathrm{C}-$ & Désinvolte & O- & Ethnocentrique \\
G- & Incivil & E- & Replié sur soi & G- & Solitaire & O- & Routinier \\
E+ & Inébranlable & E- & Distant & $\mathrm{N}-$ & Hargneux & G+ & Dévoué \\
$\mathrm{N}+$ & Zen & $\mathrm{C}+$ & Pointilleux & $\mathrm{N}+$ & Self-contrôle & E- & Secret \\
E+ & Passionné & $\mathrm{C}+$ & Persévérant & $\mathrm{C}-$ & Faible & $\mathrm{N}+$ & Paisible \\
$\mathrm{O}-$ & Conservateur & $\mathrm{E}+$ & Actif & $\mathrm{O}+$ & Inventif & $\mathrm{N}-$ & Soucieux \\
\hline
\end{tabular}

Répartition par dimension : $\mathrm{E}$ : extraversion, énergie, 0 : ouverture d'esprit, $\mathrm{C}$ : caractère consciencieux, $\mathrm{G}$ : Amabilité, N : stabilité émotionnelle.

\section{Annexe 2. Exemple de formulation des questions}

Question 1. Imaginez que vous allez bientôt être mis en contact avec quelqu'un que vous ne connaissez pas, et que la seule information que vous ayez sur cette personne est que c'est quelqu'un de tenace.

1.1. Est-ce que cette information vous est agréable, ou indifférente, ou désagréable ?

- C'est une information agréable

- Cette information me laisse indifférent(e) :

- C'est une information désagréable :

Pourquoi ?

1.2. Est-ce que cette information vous est utile, ou sans intérêt, ou gênante (et alors vous préfèreriez ne pas avoir cette information)?

- Cette information m'est utile et je suis content( e) de l'avoir

- Cette information est sans intérêt pour moi :

- Je préfèrerais ne pas avoir cette information car elle me dérange trop

Pourquoi?

\section{Annexe 3. Résultats des jugements d'agréabilité sur les dimensions extraversion et ouverture d'esprit dans leur pôle positif (données brutes et analyses statistiques)}

Annexe 3.1. Données brutes

\begin{tabular}{|c|c|c|c|c|c|c|}
\hline & \multicolumn{3}{|c|}{ Extraversion pôle positif } & \multicolumn{3}{|c|}{ Ouverture d'esprit pôle positif } \\
\hline & Femmes & Hommes & $\mathrm{F}+\mathrm{H}$ & Femmes & Hommes & $\mathrm{F}+\mathrm{H}$ \\
\hline Agréable & 182 & 183 & 365 & 233 & 223 & 456 \\
\hline Indifférent & 126 & 142 & 268 & 125 & 138 & 263 \\
\hline Désagréable & 90 & 65 & 155 & 37 & 36 & 73 \\
\hline Somme & 398 & 390 & 788 & 395 & 397 & 792 \\
\hline
\end{tabular}

NB : les sommes obtenues (788 et 792) proviennent de l'exclusion de respectivement 12 et huit réponses non fournies ou ambigües. 
Annexe 3.2. Analyses statistiques

\begin{tabular}{llll}
\hline & Femmes & Hommes & Femmes + Hommes \\
\hline Agréable & $182 / 233$, soit $\mathrm{X}^{2}=6,27, p=0,01$ & $183 / 223$, soit $\mathrm{X}^{2}=3,94, p=0,05$ & $365 / 456$, soit $\mathrm{X}^{2}=10,08, p \sim 0,00$ \\
Désagréable & $90 / 37$, soit $\mathrm{X}^{2}=22,12, p=0,01$ & $65 / 36$, soit $\mathrm{X}^{2}=8,33, p \sim 0,00$ & $155 / 73$, soit $\mathrm{X}^{2}=29,49, p \sim 0,00$ \\
\hline
\end{tabular}

Ainsi, sur le pôle positif des dimensions extraversion et ouverture d'esprit, les réponses « agréable » sont systématiquement plus fréquentes pour les informations concernant l'ouverture d'esprit que pour celles portant sur l'extraversion (avec des résultats inverses pour les réponses « désagréable »).

\section{Références}

Auzoult, L., Abdellaoui, S., 2008. Valorisation des réponses rationnelles dans la théorie expérientielle rationnelle du soi. In: Communication présentée au XII Congrès international de psychologie sociale de langue française. Université de IASI, Roumanie.

Beauvois, J.L., 1976. Problématiques des conduites sociales d'évaluation. Connexions 19, 7-30.

Beauvois, J.L., 1990. L'acceptabilité sociale et la connaissance évaluative. Connexions 56, 7-16.

Beauvois, J.L., 1995. La connaissance des utilités sociales. Psychol. Fr. 40, 375-388.

Beauvois, J.L., Dubois, N., et Peeters, G., 1999. L'évaluation personnologique. In: Beauvois, J.L., Dubois, N., Doise, W. (Eds.), La construction sociale de la personne. PUG, Grenoble, pp. 259-279.

Caprara, G.V., Barbaranelli, C., Borgogni, L., 1997. Alter Ego; les 5 facteurs fondamentaux de la personnalité. E.A.P, Paris.

Gangloff, B., 2003. D’une nature personnologique à une nature normative des 5 facteurs du Big Five. Cahiers Psychol. Pol (4).

Gangloff, B. et Mazilescu, A., 2007. Social desirability and utility of the so-called descriptive terminology of others. 5th International Conference of Applied Psychology. Timisoara.(Roumanie).

Le Barbenchon, E., Cambon, L., Lavigne, F., 2005. Désirabilité et utilité sociale de 308 adjectifs de personnalité et 297 professions. L'année psychologique 105, 307-322.

Pasquier, D., 2007. Evaluation de la personnalité à l'aide des questionnaires auto-descriptifs : approche critique du postulat de la stabilité de l'image de soi. Thèse de doctorat en psychologie. Université de Rouen, novembre 2007.

Peeters, G., 1986. Good and evil as softwares of the brain: on psychological immediates underlying the metaphysical ultimates. Interdisciplinary studies in the philosophy of understanding 9, 210-231. 